\title{
Rapid Response and Code Events in Hospitalized Children on Home Mechanical Ventilation
}

\author{
Sheila S Kun, Alexis D Deavenport-Saman, Thomas G Keens, Sally L Davidson Ward, and \\ Glenn S Takata
}

\begin{abstract}
BACKGROUND: There is limited knowledge of rapid-response (RR) events and code events for children receiving home mechanical ventilation (HMV) via a tracheostomy in a non-ICU respiratory care unit. The purpose of this study was to describe the demographic and clinical factors leading to deterioration among these children and to identify the incidence and outcomes following rapid-response and code events. METHODS: A retrospective review was conducted on hospitalized HMV children who had RR/code events in a non-ICU respiratory care unit. RESULTS: There were a total of $50 \mathrm{RR}$ events, and the primary clinical problem was acute respiratory distress, with 27 subjects $(54 \%)$ needing ventilator adjustments. Twenty (40\%) RR events occurred among children who were awaiting initial home discharge. Of 18 total code events, $7(39 \%)$ children needed a tracheostomy-related intervention. There were $10(56 \%)$ codes among children on mechanical ventilation awaiting initial home discharge. Children on HMV had 8.73 RR events per 1,000 patient days, whereas all other hospitalized children had 4.61 RR events per 1,000 patient days. In addition, children on HMV had 3.14 codes per 1,000 patient days, whereas all other hospitalized children had 0.74 codes per 1,000 patient days. All children were discharged from the hospital, and no deaths were associated with RR/code events for the index hospitalization. CONCLUSIONS: The overall incidence of RR/code events in children on HMV was higher than among non-HMV hospitalized children. Children on HMV preparing for their initial hospital discharge had the greatest number of RR/code events. The most prevalent interventions among children with $R R$ events were ventilator setting adjustments, and among children with codes the most frequent actions were tracheostomy-related interventions. Developing strategies to predict risk factors for $\mathrm{RR} / \mathrm{code}$ events may help decrease harm among children on HMV. Key words: home mechanical ventilation; rapid-response event; code event; pediatrics; incidence rate; hospitalized children; non-ICU respiratory care unit. [Respir Care 2019;64(12):1461-1468. (C) 2019 Daedalus Enterprises]
\end{abstract}

\section{Introduction}

Discharging children on mechanical ventilation via tracheostomy to their homes is no longer a novel approach to the management of chronic respiratory failure. An esti-

\footnotetext{
Dr Keen, Dr Ward, and Ms Kun are affiliated with the Division of Pulmonology, and Drs Deavenport-Saman and Takata are affiliated with the Division of General Pediatrics, Children's Hospital of Los Angeles, California.
}

Ms Kun presented a version of this article at the 14th International Conference on Home Mechanical Ventilation, held March 26-28, 2015, in Lyon, France.

The authors have disclosed no conflicts of interest. mated 8,000 children with chronic respiratory failure in the United States are on home mechanical ventilation (HMV). ${ }^{1}$ Although receiving mechanical ventilation in the home setting has been available for years, this remains one of the most demanding, high-risk, technology-dependent life support treatments for children. ${ }^{2}$ Over the past 20 years, the mortality rate of children on HMV has remained high, ranging from $20.6 \%$ to $27.5 \% .^{1,2}$

\footnotetext{
Correspondence: Sheila S Kun RN MSc, Children's Hospital of Los Angeles, 4650 Sunset Blvd, Los Angeles, California 90027. E-mail: skun@chla.usc.edu.
}

DOI: $10.4187 /$ respcare.06402 
In 1998, Ambrosio et $\mathrm{al}^{3}$ reported on the safety of clinically stable ventilator-dependent children in a respiratory care unit with an ICU transfer rate of 2.7 per 1,000 patient-days. This rate was not significantly different from that for non-ventilator-dependent children

\section{See the Related Editorial on Page 1598}

(3.3 per 1,000 patient-days). This study was, however, conducted before the development of the rapid-response (RR) team. The bedside staff detect early warning signs of potential instability and call the RR team to assist with further assessment and management, including intervention before cardiac or respiratory arrest or other serious consequences occur. Hence, the outcome data were limited to the incidence of unexpected ICU transfers and mortality rates without the benefit of earlier interventions. The concept of having a RR team of clinicians who bring critical care expertise to the bedside was first developed at Liverpool Hospital in Sydney, Australia, in 1990; they theorized that early detection of warning signs would provide an opportunity to intervene and prevent cardiac or respiratory arrest. ${ }^{4}$

There is mixed evidence on the efficacy of pediatric RR teams, particularly for the reduction in hospital mortality. ${ }^{5}$ Several studies demonstrate that the use of RR teams has been associated with improved outcomes such as reductions in rates of respiratory arrest and code events ${ }^{6-9}$; meta-analyses have indicated that RR teams are associated with reduced rates of cardiorespiratory arrest outside of the ICU and reduced mortality in both adult and pediatric populations. ${ }^{10-12}$

In children, implementation of a RR team was associated with a $37.7 \%$ reduction in rates of cardiorespiratory arrest outside of the ICU, and a $21.4 \%$ reduction in hospital mortality rates. ${ }^{10} \mathrm{RR}$ event triggers can include parameters of clinical deterioration such as the Pediatric Early Warning Score, which identifies children at risk of cardiorespiratory arrest. ${ }^{13}$ This may provide a forewarning time of $>11 \mathrm{~h}$ and may avert a code. ${ }^{14,15}$ Younger children with a chronic disease and various comorbidities may have benefitted from planning, placement, and early ICU intervention afforded by RR events. ${ }^{6}$ Previous studies have examined RR events in pediatric in-patient units by examining child diagnoses, interventions, and post-intervention clinical outcomes and placement, ${ }^{16}$ but there have not been any studies focusing on children on HMV. The incidence, demographic information, clinical presentation, and outcomes of $\mathrm{RR} /$ code events among children on HMV in a non-ICU respiratory care unit are not well known. The purpose of our study was thus to describe the demographic and clinical factors leading to deterioration among children on HMV in a non-ICU respiratory care unit and to identify the incidence and outcomes following RR and code events.

\section{QUICK LOOK}

\section{Current knowledge}

The incidence of rapid-response (RR) and code events among hospitalized children has been established, but not for children on home mechanical ventilation (HMV) in a respiratory care unit. There is limited knowledge of children's demographic and clinical characteristics associated with RR and code events, which could help in the development of strategies to decrease harm.

\section{What this paper contributes to our knowledge}

In a tertiary care setting, the incidence of RR/code events and transfers to ICUs were higher among children on HMV in a respiratory care unit than other children. Children on HMV awaiting initial home discharge were at particular risk for RR and code events. RR events were primarily triggered by respiratory signs and symptoms and agitation, followed by interventions addressing ventilator issues. Codes were primarily triggered by tracheostomy-related complications and were followed by tracheostomy-related interventions.

\section{Methods}

\section{Design}

A retrospective descriptive analysis was conducted on children who required positive-pressure ventilation via tracheostomy (ie, home mechanical ventilation) and who were hospitalized in a non-ICU respiratory care unit and received $\mathrm{RR} /$ code events. Data were obtained from an RR/code event database from January 2010 to September 2013 and from a chart review. The study was approved by the institutional review board of Children's Hospital of Los Angeles.

\section{Setting and Participants}

This study took place at a tertiary care freestanding children's hospital in Los Angeles, which cares for one of the largest groups of children on HMV in the western United States, with an average of $>200$ children on HMV receiving care each year. Children on HMV were included in this retrospective study if they had experienced an RR or code event on a portable home ventilator while managed in a non-ICU respiratory care unit. Many hospitals in the United States admit children on HMV exclusively to the ICU because physicians are often reluctant to transfer ventilator-dependent patients outside of the ICU. ${ }^{3}$ Our hospital, however, admits stable children on HMV to a non-ICU respiratory care unit. At the time of this study, the unit had a total HMV capacity 


\section{Emergency Response in Pediatric Home Mechanical Ventilation}

of 16 patients. This included children hospitalized for intercurrent illnesses, for elective and nonelective surgical procedures, and those who were being readied for initial home discharge. The attending physician for the non-ICU respiratory care unit was the rotating pulmonologist for the in-patient service, with a pulmonary fellow in attendance. They were accompanied either by an attending hospitalist or by a pediatric resident physician assigned to oversee children on HMV. The night shift was covered by a pediatric resident, with a pulmonary fellow and pulmonary attending physician on call. There were at least 2 respiratory practitioners assigned to the unit to provide respiratory assessment and support. The nurse:patient ratio for children receiving HMV in the non-ICU respiratory care unit was 1:2, whereas in the ICU this ratio is $1: 1$. The respiratory therapist:patient ratio for children receiving HMV in the non-ICU respiratory care was $1: 8$ versus $1: 3$ in the ICU. Respiratory care unit placement for stable children on HMV was established more than 20 years ago at our hospital; bedside staff were not trained in critical care management.

Created in 1997, the hospital's RR team is a group of health care professionals with critical care expertise that is prepared to respond to patients at the first signs of clinical deterioration. ${ }^{17-19}$ The team's goal is to stabilize a child's condition before a cardiac or respiratory arrest or another adverse event occurs. The RR team is intended to supplement current practice patterns and the chain of command while enabling prompt assistance to the child. The RR team is composed of a pediatric ICU fellow or attending, the lead respiratory therapist, a pediatric ICU nurse, and a house nursing supervisor. The target response time from calling the operator to at least one RR team member arriving at the bedside is $\sim 10 \mathrm{~min}$. If the child's condition deteriorates at any time due to cardiac or respiratory arrest, the Code Blue team is activated immediately. RR teams can be activated by physicians, respiratory therapists, nurses, or parents to initiate the process of immediate assessment for potential deterioration of the child's condition.

The Code Blue team responds to code events that are called for emergency situations. Basic life support is immediately initiated for all patients having a cardiac or respiratory arrest, unless there is a written do-not-resuscitate order. Two code teams, trained and certified based on national guidelines, service the campus. ${ }^{20}$ The Code Blue team includes a physician, an intravenous infusion nurse, a house nursing supervisor, an ICU nurse, a respiratory therapist, a social worker, and a chaplain.

\section{Data Collection and Measures}

A chart review was conducted to gather data on demographic characteristics, clinical circumstances prompting $\mathrm{RR} /$ code events, outcomes of RR/code events, RR/code interventions, outcomes of ICU transfer, and discharge status.
Demographic and clinical variables included age, gender, whether children had $\geq 2 \mathrm{RR} /$ code events within the study period, shift, day of the week, and season. Season was defined as winter (October-March) and non-winter (April-September). Hospital census data included information on whether children were hospitalized inside or outside of the ICU. The rate of RR/codes events was defined as RR events per 1,000 patient-days and code events per 1,000 patient-days. The ICU transfer rate was defined as the rate of those with RR/codes events who were transferred to the ICU per 1,000 patientdays. All hospitalized children on HMV in the respiratory care unit were compared to all non-HMV hospitalized children not in the ICU over the same period.

\section{Statistical Analysis}

SPSS 17 (IBM, Armonk, New York) was used to run descriptive analyses on demographic characteristics of the children and their RR/code events. Rates of RR/code events and ICU transfers with 95\% CIs were also calculated for HMV and non-HMV children.

\section{Results}

\section{Rapid-Response Events}

RR Event Demographic and Clinical Factors. There were a total of 50 RR events among 26 children on HMV in a respiratory care unit. The mean \pm SD age of children with RR events was $3.58 \pm 4.36 \mathrm{y}$, and the median (interquartile range) age was $1.42(0.37-2.47)$ y. Twentyeight RR events (56\%) involved male children. There were 35 RR events (70\%) among 11 children who each experienced $\geq 2 R R$ events, including the initial RR event (Table 1). The primary diagnoses associated with RR events included chronic lung disease (31 [62\%]), ventilatory muscle weakness (12 [24\%]), and congenital central hypoventilation syndrome (7 [14\%]). There were 20 (40\%) HMV RR events among children being readied for initial home discharge, $9(18 \%)$ in children who had been discharged home for $<3$ months, and $21(42 \%)$ in those who had been discharged for $>3$ months.

Of the 50 reported RR events, $26(52 \%)$ of the children presented with worsening oxygen desaturation, 18 (36\%) with carbon dioxide retention, 15 (30\%) demonstrated agitation, and 14 (28\%) with increased dyspnea (Fig. 1). Tracheostomy-related problems included thick tracheal secretions with risk for airway occlusion in $11(22 \%)$ children and decannulation in $1(2 \%)$ child. Other signs resulting in RR events included lethargy (8 [16\%]), tachycardia (6 [12\%]), fever (5 [10\%]), and blood pressure changes (4 [8\%]). Some children had more than one presenting symptom. There were no RR events among chil- 
Table 1. Demographic and Clinical Factors Related to RR and Code Events in Hospitalized Children on HMV

\begin{tabular}{|c|c|c|}
\hline & RR Events & Code Events \\
\hline \multicolumn{3}{|l|}{ Age group } \\
\hline$<1 \mathrm{y}$ & $8(16)$ & $6(33.3)$ \\
\hline $1-2 y$ & $21(42)$ & $6(33.3)$ \\
\hline $3-4 y$ & $12(24)$ & $1(5.6)$ \\
\hline $5-6 y$ & $6(12)$ & 0 \\
\hline$\geq 7 y$ & $3(6)$ & $5(27.8)$ \\
\hline \multicolumn{3}{|l|}{ Gender } \\
\hline Male & $28(56)$ & $13(72.2)$ \\
\hline Female & $22(44)$ & $5(27.8)$ \\
\hline \multicolumn{3}{|l|}{$\geq 2$ events } \\
\hline No & $15(30)$ & $9(50)$ \\
\hline Yes & $35(70)$ & $9(50)$ \\
\hline \multicolumn{3}{|l|}{ Shift } \\
\hline Day & $30(60)$ & $13(72.2)$ \\
\hline Night & $20(40)$ & $5(27.8)$ \\
\hline \multicolumn{3}{|c|}{ Day of the week } \\
\hline Weekday & $39(78)$ & $15(83.3)$ \\
\hline Weekend & $11(22)$ & $3(16.7)$ \\
\hline \multicolumn{3}{|l|}{ Season } \\
\hline Non-winter & $21(42)$ & 7 (38.9) \\
\hline Winter & $29(58)$ & $11(61.1)$ \\
\hline \multicolumn{3}{|c|}{$\begin{array}{l}\text { Data are presented as } n(\%) \text {. RR events: } n=50 \text {; Code events: } n=18 . \\
\text { RR }=\text { rapid-response } \\
\text { HMV }=\text { home mechanical ventilation }\end{array}$} \\
\hline
\end{tabular}

dren who were being observed after anesthesia or sedation for surgery or other procedures.

The RR interventions for the HMV children included a variety of strategies, the most common being adjusting ventilator settings and secretion clearance (Fig. 2). There were $27(54 \%)$ RR events that required assessment of respiratory needs and adjustment of ventilator settings, such as rate or pressure changes to allow for better gas exchange; supplementary oxygen was titrated upward in 22 of these 27 ventilator-adjustment responses. Secretion clearance therapy was needed in $24(48 \%)$ children, with the use of suctioning in 21 (42\%) or a cough-assist device in $3(6 \%)$ children. Chest radiograph was obtained in $20(40 \%)$ children for cardiopulmonary assessment, and bronchodilators were used in $11(22 \%)$ children. Tracheostomy tube replacement was performed in 10 (20\%) children, and $1(2 \%)$ child needed chest tube placement. Other intervening strategies included administering sedation to allow relaxation and better synchrony of ventilation for $16(32 \%)$ of children. Refer to Figure 2 for the less frequent interventions.

RR Event Incidence Rates, ICU Transfer Rates, and Outcomes. Children on HMV had 50 RR events out of 5,729 patient-days in the respiratory care unit, which was a rate of 8.73 RR events (95\% CI 8-9.46) per 1,000 patient-days, while all other hospitalized children had 1,176 RR events out of 255,086 patient-days, which was a rate of $4.61 \mathrm{RR}$ events (95\% CI $4.53-4.69$ ) per $1,000 \mathrm{pa}-$ tient-days (Table 2). A total of 27 of $50(54 \%)$ children on HMV with an RR event were transferred to the ICU, whereas 507 of 1,176 (43\%) non-HMV hospitalized children with an RR event were transferred to the ICU. There was no mortality associated with RR events, and all children were discharged home.

\section{Code Events}

Code Demographics and Clinical Factors. There were a total of 18 code events among 12 children on HMV. The mean $\pm \mathrm{SD}$ age of children with code events was

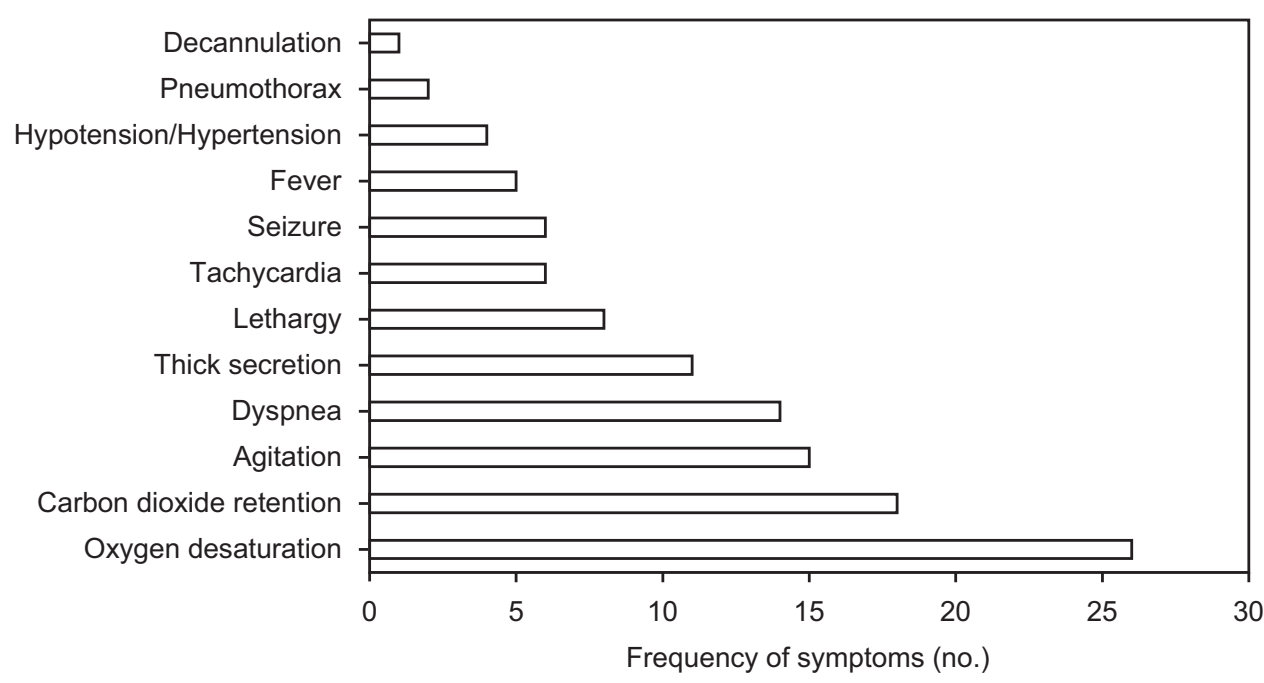

Fig. 1. Frequency of presenting symptoms of rapid-response events. Total events $=50$. 


\section{Emergency Response in Pediatric Home Mechanical Ventilation}

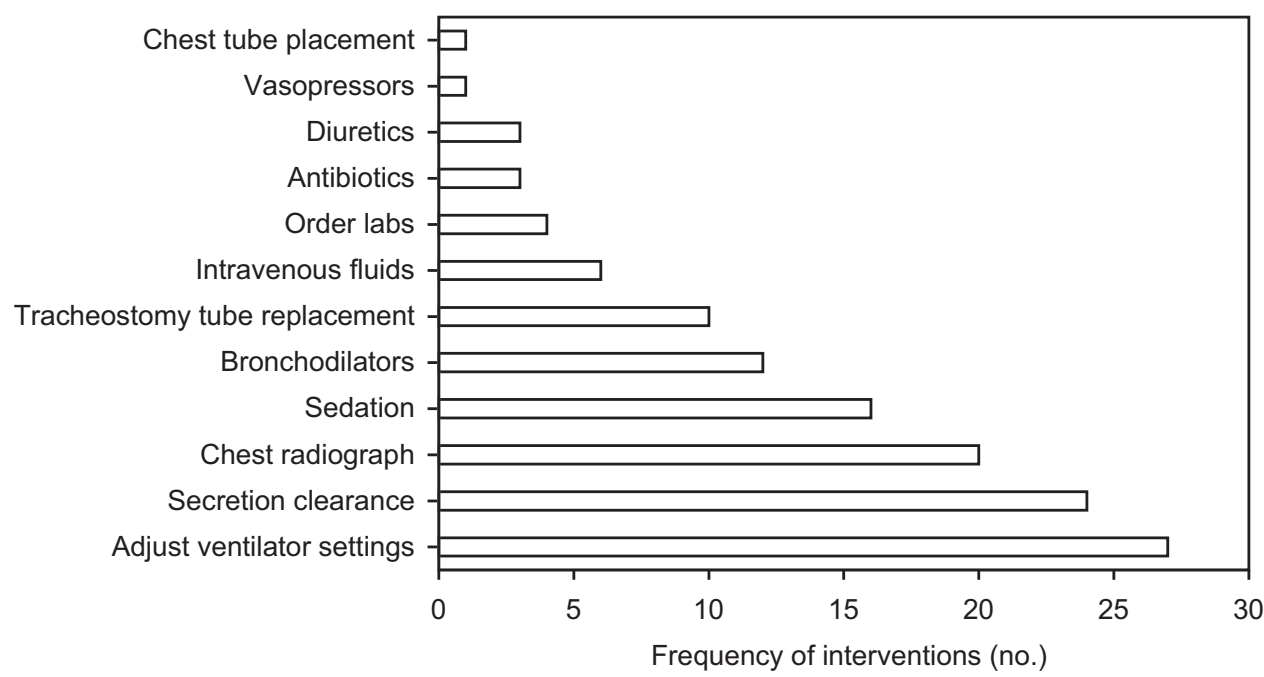

Fig. 2. Frequency of interventions for rapid-response events. Total events $=50$.

Table 2. Rates of RR Events and ICU Transfers Among HMV and Non-HMV Children

\begin{tabular}{|c|c|c|c|}
\hline RR Events & $\begin{array}{l}\text { Children on HMV } \\
\quad(n=50)\end{array}$ & $\begin{array}{l}\text { Non-HMV Hospitalized } \\
\text { Children }(n=1,176)\end{array}$ & $\begin{array}{l}\text { All Children } \\
(n=1,226)\end{array}$ \\
\hline Patient days, no. & 5,729 & 255,086 & 260,815 \\
\hline Rate of RRs per 1,000 patient days* & $8.73(8-9.46)$ & $4.61(4.53,4.69)$ & $4.7(4.62,4.78)$ \\
\hline RR Event Initiated Transfer to ICU & $\begin{array}{l}\text { Children on HMV } \\
\quad(n=27)\end{array}$ & $\begin{array}{l}\text { Non-HMV Hospitalized Children } \\
\qquad(n=507)\end{array}$ & $\begin{array}{l}\text { All Children } \\
(n=534)\end{array}$ \\
\hline Patient days, no. & 5,729 & 255,086 & 260,815 \\
\hline ICU transfer rate per 1,000 patient days* & $4.71(4.16-5.26)$ & $1.99(1.94-2.04)$ & $2.05(2-2.1)$ \\
\hline $\begin{array}{l}\text { * Presented as the rate }(95 \% \mathrm{CI}) \\
\mathrm{RR}=\text { rapid-response } \\
\mathrm{HMV}=\text { home mechanical ventilation }\end{array}$ & & & \\
\hline
\end{tabular}

$5.84 \pm 7.69 \mathrm{y}$, and the majority of code events (13 [72.2\%]) involved male children. There were 9 code events $(50 \%)$ among 3 children who each experienced a total of $\geq 2$ code events, including the initial code event. The primary diagnoses associated with children on HMV who had codes were ventilatory muscle weakness $(9[50 \%])$, chronic lung disease $(8[44.4 \%])$, and congenital central hypoventilation syndrome $(1[5.6 \%])$. The presenting symptoms associated with codes in children were tracheostomy-related in $7(38.9 \%)$ and required tracheostomy-related interventions. Six (33.3\%) children had abnormalities of gas exchange and needed ventilator adjustments, $2(11.1 \%)$ demonstrated agitation and required sedation, $2(11.1 \%)$ had respiratory distress, and 1 (5.6\%) child had bradycardia, with an underlying cardiac diagnosis. There were no code events among children who were being observed after anesthesia or sedation for surgery or other procedures. Of the 18 codes, $17(94.4 \%)$ were for re- spiratory arrests and $1(5.6 \%)$ for cardiac arrest. There were $10(55.6 \%)$ codes among children on HMV awaiting initial home discharge; no codes occurred among those who were discharged home for $<3$ months, and $8(44.4 \%)$ codes occurred among those who had been discharged for $>3$ months.

Code Event Incidence Rates, ICU Transfer Rates, and Outcomes. Children on HMV in the respiratory care unit had 18 code events out of 5,729 patient-days, which was a rate of 3.14 code events (95\% CI 2.69-3.59) per 1,000 patient-days, while all other hospitalized children had 188 code events out of 255,086 patient-days, which was a rate of 0.74 code events (95\% CI 0.71-0.77) per 1,000 patient-days (Table 3). All 18 children on HMV with a code event were transferred to the ICU, whereas 45 of 188 (23.9\%) non-HMV hospitalized children with a code event were transferred to the ICU. There were no 
Table 3. Rates of Code Events and ICU Transfers Among HMV and Non-HMV Children

\begin{tabular}{lccc}
\hline \hline \multicolumn{1}{c}{ Code Event } & $\begin{array}{c}\text { Children on HMV } \\
(n=18)\end{array}$ & $\begin{array}{c}\text { Non-HMV Hospitalized Children } \\
(n=188)\end{array}$ & $\begin{array}{c}\text { All Children } \\
(n=206)\end{array}$ \\
\hline $\begin{array}{l}\text { Patient days, no. } \\
\text { Rate of codes per 1,000 patient days* }\end{array}$ & $\begin{array}{c}5,729 \\
(2.69-3.59)\end{array}$ & $\begin{array}{c}255,086 \\
0.74(0.71-0.77)\end{array}$ & $0.79(0.76-0.82)$ \\
\hline Code Event Initiated Transfer to ICU & $\begin{array}{c}\text { Children on HMV } \\
(n=18)\end{array}$ & $\begin{array}{c}\text { Non-HMV Hospitalized Children } \\
(n=45)\end{array}$ & $\begin{array}{c}\text { All Children } \\
(n=63)\end{array}$ \\
\hline $\begin{array}{l}\text { Patient days, no. } \\
\text { ICU transfer rate per 1,000 patient days* }\end{array}$ & $\begin{array}{c}5,729 \\
\text { * }\end{array}$ & $0.18(0.16-0.2)$ & 260,815 \\
* Presented as the rate $(95 \%$ CI). & & & $0.24(0.22-0.26)$ \\
HMV = home mechanical ventilation & & & \\
\hline
\end{tabular}

deaths associated with codes, and all children were discharged home.

\section{Discussion}

To our knowledge, this study is the first to provide data on the demographic and clinical factors leading to deterioration among children on HMV via a tracheostomy in a respiratory care unit and with identification of the incidence and outcomes following RR/code events. The overall incidence of RR/code events for children on HMV was greater than that for other children hospitalized outside of ICUs. Factors such as the compromised respiratory state among children on HMV and the technological complexities and challenges of caring for children on HMV may have contributed to the higher incidence of RR/code events. This information may help to develop strategies to prevent RR/codes among children on HMV admitted to a respiratory care unit in the future. Results highlighted the importance of focusing on children on HMV awaiting initial home discharge. During this transition period, ventilator setting adjustments were often needed to establish optimum home ventilator settings. Some children may not have tolerated the initial home ventilator settings, which may have led to critical changes in their respiratory status.

Children on HMV being readied for initial hospital discharge with worsening gas exchange or tracheostomy-related complications were more likely to have RR/code events. There may be several root causes why children on HMV awaiting initial home discharge were at greater risk for RR/code events. At our hospital, all children placed on HMV are initially in the ICU and are then transferred to the respiratory care unit after they have demonstrated stability on a portable home ventilator for a period of days. Portable home ventilators do not offer as many features or modalities as those used in ICUs. Unlike the ICU, which has higher ratios of respiratory therapists and nurses to patients, as well as protocols and standing orders to monitor gas exchange and to make frequent ventilator changes, in the respiratory care unit there are no protocols or standing orders that allow for frequent changes in ventilator settings by respiratory therapists or nurses, although ventilators are checked every $4 \mathrm{~h}$. If there is any major change in a child's respiratory status, the immediate response is to call a RR event and to notify the pulmonologist. Therefore, although stable on transfer from the ICU, once in the respiratory care unit days or weeks of adjustment may be necessary to achieve optimum settings on the portable home ventilator. Thus, the children in this study being readied for initial hospital discharge may have had more RR events and needed ventilator adjustments in response to the transition to a home ventilator.

Decreasing potential RR events during the transitional period in preparation for initial home discharge from the respiratory care unit is difficult because these children experience an array of clinical challenges. For example, they may have increased respiratory load, chest wall anomalies, abdominal wall defects, and central drive, ventilatory muscle, and airways defects. Developing standardized policies and protocols to optimize their ventilator needs during the transitional period might be difficult because each child's respiratory demands vary. Treatment plans are customized to the individual's needs; thus determining the best settings for all new children on HMV is difficult. Home ventilators, compared with those used for acute care, are simple and not as versatile; their features are more limited when ventilating patients with complex respiratory conditions. During the transition from hospital units to home ventilators, RR events are therefore more likely to occur.

The higher incidence of RR/code events among children on HMV compared to hospitalized children not on HMV was expected. American Thoracic Society guidelines for home mechanical ventilation recommends intensive training for care providers and close monitoring of children on $\mathrm{HMV}$, recognizing that children who require chronic invasive ventilation at home experience numerous challenges. ${ }^{21,22}$ The difference in ICU transfer rates between 


\section{Emergency Response in Pediatric Home Mechanical Ventilation}

HMV and non-HMV hospitalized children was lower for those experiencing a RR event than those experiencing a code; these differences may have been due to chance and the small number of codes in this study, and so these differences may not be clinically important. The higher rate of RR/code events among children on HMV suggests that greater vigilance is warranted in anticipating the possible deterioration of these children in the respiratory care unit, particularly among those awaiting initial home discharge. Interestingly, postoperative care and recovery from anesthesia and sedation were not associated with RR/code events. Learning from RR events may help prevent adverse events. ${ }^{23}$ Although not addressed by the data in our study, a longer period of stabilization of children on HMV awaiting initial home discharge in the ICU before transferring to a respiratory care unit merits further study.

These findings have had 2 significant applications in practice at the study site. First, these results were used to better prepare and train our staff caring for children on HMV in the respiratory care unit. After recognizing that the most common early presenting symptoms of deterioration were related to respiratory changes in children making the transition to the home ventilator, the HMV respiratory care unit formed a task force to address these problems. Second, the relatively low incidence of RR/code events and an absence of mortality during the index hospitalization suggested that children on HMV can be cared for safely in a lower intensity of care than the ICU; however, because children on HMV had a higher incidence of $\mathrm{RR} /$ code events than other children, hospitalization at an intermediate level of care (eg, in a respiratory care unit), rather than the regular in-patient unit, seemed warranted.

Study limitations include the retrospective analysis of data and the availability of data in the RR event database and medical records. In a future study, we hope to consider whether the time of discharge from the ICU is associated with RR/code events. In addition, many children had multiple comorbidities that might have contributed to the $\mathrm{RR} /$ code events, and the presenting symptoms of deterioration might not capture all the underlying causes. A larger study would be needed to pinpoint which co-existing conditions played a role in RR/code events. The findings for $\mathrm{RR} /$ code events for day of the week and shift may not be clinically important, given the small sample sizes. This study only considered children on HMV on portable home ventilators, which may not be generalizable to other hospital settings. Finally, although we care for one of the largest groups of children on HMV in the western United States, data were from one children's hospital and may not be representative of children on HMV in other hospitals because many hospitals do not manage these children outside of ICU settings.

\section{Conclusions}

The overall incidence of RR/code events was higher for children on HMV in a respiratory care unit compared to all other hospitalized children. The relatively low incidence of RR/code events and the absence of mortality during the index hospitalization suggests that children on HMV can be cared for safely in a respiratory care unit. RR events were primarily triggered by respiratory signs and symptoms and agitation, followed by interventions addressing ventilator issues. Codes were primarily triggered by tracheostomy-related complications and followed by tracheostomyrelated interventions. Children on HMV awaiting initial home discharge transitioning from the hospital to a home ventilator require continuous and vigilant monitoring. Developing future strategies to predict HMV children who are at risk for $\mathrm{RR} /$ code events may help to decrease harm.

\section{REFERENCES}

1. Boroughs D, Dougherty JA. Decreasing accidental mortality of ventilator-dependent children at home: a call to action. Home Health Nurse 2012;30(2):103-111.

2. Edwards JD, Kun SS, Keens TG. Outcomes and causes of death in children on home mechanical ventilation via tracheostomy: an institutional and literature review. J Pediatr 2010;157(6):955-959.

3. Ambrosio IU, Woo MS, Jansen MT, Keens TG. Safety of hospitalized ventilator-dependent children outside of the intensive care unit. Pediatrics 1998;101(2):257-259.

4. Frost SA, Chapman A, Aneman A, Chen J, Parr MJ, Hillman K. Hospital outcomes associated with introduction of a two-tiered response to the deteriorating patient. Crit Care Res 2015;17(2):77-82.

5. Joffe AR, Anton NR, Burkholder SC. Reduction in hospital mortality over time in a hospital without a pediatric medical emergency team: limitations of before-and-after study designs. Arch Pediatr Adolesc Med 2011;165(5):419-423.

6. Wang GS, Erwin N, Zuk J, Henry DB, Dobyns EL. Retrospective review of emergency response activations during a 13-year period at a tertiary care children's hospital. J Hosp Med 2011;6(3):131-135.

7. Hanson CC, Randolph GD, Erickson JA, Mayer CM, Bruckel JT, Harris BD, Willis TS. A reduction in cardiac arrests and duration of clinical instability after implementation of a paediatric rapid response system. Qual Saf Health Care 2009;18(6):500-504.

8. Hunt EA, Zimmer KP, Rinke ML, Shilkofski NA, Matlin C, Garger $\mathrm{C}$, et al. Transition from a traditional code team to a medical emergency team and categorization of cardiopulmonary arrests in a children's center. Arch Pediatr Adolesc Med 2008;162(2):117-122.

9. Sharek PJ, Parast LM, Leong K, Coombs J, Earnest K, Sullivan J, et al. Effect of a rapid response team on hospital-wide mortality and code rates outside the ICU in a children's hospital. JAMA 2007; 298(19):2267-2274.

10. Chan PS, Jain R, Nallmothu BK, Berg RA, Sasson C. Rapid response teams: a systematic review and meta-analysis. Arch Intern Med 2010; 170(1):18-26.

11. Winters BD, Weaver SJ, Pfoh ER, Yang T, Pham JC, Dy SM. Rapid-response systems as a patient safety strategy: a systematic review. Ann Intern Med 2013;158(5 Pt 2):417-425.

12. Maharaj R, Raffaele I, Wendon J. Rapid response systems: a systematic review and meta-analysis. Crit Care 2015;19:254.

13. Parshuram CS, Duncan HP, Joffe AR, Farrell CA, Lacroix JR, Middaugh $\mathrm{KL}$, et al. Multicentre validation of the bedside paediatric 


\section{Emergency Response in Pediatric Home Mechanical Ventilation}

early warning system score: a severity of illness score to detect evolving critical illness in hospitalised children. Crit Care 2011; 15(4):R184.

14. Akre M, Finkelstein M, Erickson M, Liu M, Vanderbilt L, Billman G. Sensitivity of the pediatric early warning score to identify patient deterioration. Pediatrics 2010;125(4):e763-e769.

15. Bonafide CP, Roberts KE, Priestley MA, Tibbetts KM, Huang E, Nadkarni VM, Keren R. Development of a pragmatic measure for evaluating and optimizing rapid response systems. Pediatrics 2012; 129(4):e874-e881.

16. ul-Haque A, Saleem AF, Zaidi S, Haider SR. Experience of pediatric rapid response team in a tertiary care hospital in Pakistan. Indian J Pediatr 2010;77(3):273-276.

17. Buist MD, Jarmolowski E, Burton PR, Bernard SA, Waxman BP, Anderson J. Recognising clinical instability in hospital patients before cardiac arrest or unplanned admission to intensive care. A pilot study in a tertiary-care hospital. Med J Aust 1999;171(1):22-25.

18. Franklin C, Mathew J. Developing strategies to prevent in hospital cardiac arrest: analyzing responses of physicians and nurses in the hours before the event. Crit Care Med 1994;22(2):244-247.
19. Tibballs J, Kinney S, Duke T, Oakley E, Hennessy M. Reduction of paediatric in-patient cardiac arrest and death with a medical emergency team: preliminary results. Arch Dis Child 2005;90(11):11481152.

20. American Heart Association. Part 6: Advanced cardiovascular life support: section 1: introduction to ACLS 2000: overview of recommended changes in ACLS from the Guidelines 2000 Conference. Circulation 2000;102:I86-I89.

21. Sherman JM, Davis S, Albamonte-Petrick S, Chatburn RL, Fitton C, Green C, et al. Care of the child with a chronic tracheostomy. Am J Respir Crit Care Med 2000;161(1):297-308.

22. Sterni LM, Collaco JM, Baker CD, Carroll JL, Sharma GD, Brozek JL, et al. An official American Thoracic Society clinical practice guideline: pediatric chronic home invasive ventilation. Am J Respir Crit Care Med 2016;193(8):e16-e35.

23. Amaral AC, McDonald A, Coburn NG, Xiong W, Shojania KG, Fowler RA, et al. Expanding the scope of critical care rapid response teams: a feasible approach to identify adverse events. A prospective observational cohort. BMJ Qual Saf 2015;24(12):764-768.

This article is approved for Continuing Respiratory Care Education credit. For information and to obtain your CRCE

(free to AARC members) visit www.rcjournal.com 